\title{
Generalidades de las venas varicosas en miembros inferiores y su tratamiento durante el embarazo: una revisión narrativa ${ }^{1}$
}

\author{
Ligia Patricia Rojas Valenciano ${ }^{2}$ \\ Henry Escobar Fonseca ${ }^{3}$ \\ Pilar Cárdenas Sánchez ${ }^{4}$ \\ Jennifer González Bermúdez ${ }^{5}$
}

INSTITUCIÓN: Universidad de Costa Rica

\begin{abstract}
RESUMEN
La enfermedad varicosa es un problema común que afecta a gran parte de la población mundial, principalmente en mujeres, agravado por una condición de embarazo. Esta revisión narrativa tiene el objetivo de conocer la evidencia científica disponible sobre aspectos diagnósticos, factores de riesgo e intervención conservadora o invasiva, incluyendo cirugía de las venas varicosas en miembros inferiores en mujeres embarazadas. La búsqueda bibliográfica se llevó a cabo en las bases de datos PubMed, Biblioteca Cochrane y EBSCO Host entre los años 2009 y 2017. Se recuperó 50 referencias bibliográficas y se seleccionó seis documentos para el análisis. Respecto del diagnóstico se lleva a cabo mediante la observación, examen directo, historia clínica y exámenes más específicos como el doppler. La síntomatología más común que se presenta es el dolor en miembros inferiores, edema, pesadez, prurito y calambres. La intervención para el tratamiento a mujeres embarazadas es la terapia conservadora, aunque solo en casos excepcionales se determinará otro tipo de tratamiento. En este proceso, la educación tiene un rol muy importante en la seguridad de la madre y el feto, cuyo abordaje debe ser dado por un equipo de profesionales en salud. Se concluye que existe controversias respecto de la patogenia de esta morbilidad durante el embarazo, por lo tanto, no se ofrece otros tratamientos distintos a los recomendados desde el año 50, de manera que hay una gran necesidad de llevar a cabo estudios robustos que demuestren cuáles son las mejores medidas profilácticas más novedosas y de tratamiento desde el inicio del embarazo, además de promover la prevención de la aparición posterior, de tal manera que esté asegurada la salud de la mujer y, consecuentemente, la de su bebé.
\end{abstract}

Palabras clave: embarazo, tratamiento-venas-varicosas, venas-varicosas

\footnotetext{
DOI: https://doi.org/10.15517/revenf.v0i35.32824

${ }^{1}$ Fecha de recibido: 10 de marzo del 2018

Fecha de aceptación: 24 de mayo del 2018

${ }^{2}$ Enfermera obstetra. Doctora en Educación. Catedrática de la Universidad de Costa Rica. Profesora de la Escuela de Enfermería. Costa Rica. Correo electrónico: ligiarojas7@gmail.com

${ }^{3}$ Médico y cirujano. Universidad del Valle, Cali, Colombia. Especialista en Cirugía Vascular, Universidad de Costa Rica. Cirujano independiente. Exprofesor de la Facultad de Medicina de la Universidad Santiago de Cali. Colombia. Correo electrónico: medicosencali@hotmail.com

${ }^{4}$ Enfermera. Estudiante de la Maestría en enfermería ginecológica, obstétrica y perinatal de la Universidad de Costa Rica. Trabajo independiente. Costa Rica. Correo electrónico: pilicardenas28@gmail.com

${ }^{5}$ Enfermera. Estudiante de la Maestría en enfermería ginecológica, obstétrica y perinatal de la Universidad de Costa Rica. Trabajo independiente. Costa Rica. Correo electrónico: jennygo78@gmail.com
} 


\title{
Generalities regarding varicose veins in lower limbs and their treatment during pregnancy: a narrative review 1
}

\author{
Ligia Patricia Rojas Valenciano ${ }^{2}$ \\ Henry Escobar Fonseca ${ }^{3}$ \\ Pilar Cárdenas Sánchez ${ }^{4}$ \\ Jennifer González Bermúdez ${ }^{5}$
}

INSTITUTION: University of Costa Rica

\begin{abstract}
The varicose disease is a common problem that affects a large part of the world population in greater percentage of women, added to its condition the presence of pregnancy. This narrative review has the objective of knowing the available scientific evidence on diagnostic aspects, risk factors and conservative or invasive intervention including surgery of varicose veins in lower limbs in pregnant women. The bibliographic search was carried out in PubMed, Cochrane Library and EBSCO Host databases between 2009 and 2017. Fifty bibliographical references were retrieved and six documents were selected for analysis. Regarding the diagnosis is carried out through observation, direct examination, clinical history, more specific examinations such as Doppler. The most common symptom presented is a pain in the lower limbs, oedema, heaviness, pruritus and cramps. The intervention for the treatment of pregnant women is conservative therapy, only in exceptional cases will be determined another type of treatment. Education plays a very important role in the safety of the mother and the fetus. The approach to these women must be given by a team of health professionals. It is concluded that there are controversies regarding the pathogenesis of this morbidity during pregnancy and, therefore, no treatments other than those recommended since year 50 are offered, so there is a great need to carry out robust studies that show which are the best new prophylactic measures and treatment from the beginning of pregnancy, in addition to promoting the prevention of subsequent appearance, in such a way that the health of the woman and consequently that of her baby is ensured.
\end{abstract}

Keywords: pregnancy, varicose-vein treatment, varicose veins

DOI: https://doi.org/10.15517/revenf.v0i35.32824

${ }^{1}$ Date of receipt: March 10, 2018

Date of acceptance: May 24, 2018

${ }^{2}$ Obstetrical nurse. Doctor in Education. Catedratical Professor of the School of Nursing of the University of Costa Rica. Costa Rica. Email: ligiarojas7@gmail.com

${ }^{3}$ Physician and Surgeon. University of the Valley, Cali, Colombia. Specialist in Vascular Surgery, University of Costa Rica.

Independent surgeon. Former professor at the Faculty of Medicine of the Santiago de Cali University. Colombia. E-mail: medicosencali@hotmail.com

${ }^{4}$ Nurse. Student of the Posgraduate Ginecological, Obstetrical and Perinatal Nursing of the University of Costa Rica. Independent work. Costa Rica. E-mail: pilicardenas28@gmail.com

${ }^{5}$ Nurse. Student of the Posgraduate Ginecological, Obstetrical and Perinatal Nursing of the University of Costa Rica. Independent work. Costa Rica. E-mail: jennygo78@gmail.com 


\title{
Generalidades das varizes em membros inferiores e seu tratamento durante a gravidez: uma revisão narrativa ${ }^{1}$
}

\author{
Ligia Patricia Rojas Valenciano ${ }^{2}$ \\ Henry Escobar Fonseca ${ }^{3}$ \\ Pilar Cárdenas Sánchez ${ }^{4}$ \\ Jennifer González Bermúdez ${ }^{5}$
}

Instituição: Universidade da Costa Rica

\begin{abstract}
RESUMO
A doença varicosa é um problema comum que afeta uma grande parte da população mundial em maior porcentagem para as mulheres, adicionou à sua condição a presença de gravidez. Esta revisão narrativa tem como objetivo conhecer as evidências científicas disponíveis sobre aspectos diagnósticos, fatores de risco e intervenção conservadora ou invasiva, incluindo cirurgia de varizes em membros inferiores em mulheres grávidas. A pesquisa bibliográfica foi realizada nas bibliotecas PubMed, Cochrane Library e EBSCO Host entre 2009 e 2017. Cinquenta referências bibliográficas foram recuperadas e 6 documentos foram selecionados para análise. Quanto ao diagnóstico é realizado através da observação, exame direto, história clínica, exames mais específicos, como Doppler. O sintoma mais comum apresentado é a dor nos membros inferiores, edema, peso, prurido e cólicas. A intervenção para o tratamento de mulheres grávidas é a terapia conservadora, apenas em casos excepcionais será determinado outro tipo de tratamento. A educação desempenha um papel muito importante na segurança da mãe e do feto. A abordagem dessas mulheres deve ser dada por uma equipe de profissionais de saúde. Conclui-se que há controvérsias quanto à patogenia desta morbidade durante a gravidez e, portanto, não são oferecidos outros tratamentos além dos recomendados desde o ano 50, por isso há uma grande necessidade de realizar estudos robustos que mostrem quais são as melhores medidas profiláticas novas e o tratamento desde o início da gravidez, além de promover a prevenção da aparição subseqüente, de tal forma que seja assegurada a saúde da mulher e conseqüentemente a de seu bebê.
\end{abstract}

Palavras-chave: gravidez, tratamento de veias varicosas, varizes

\footnotetext{
DOI: https://doi.org/10.15517/revenf.v0i35.32824

${ }^{1}$ Data da recepção: 10 de março de 2018

Data de aceitação: 24 de maio de 2018

${ }^{2}$ Enfermeira obstétrica. Doutor em Educação. Professor Catedratico da Universidade da Costa Rica. Professor da Escola de

Enfermagem. Costa Rica. Correio eletrônico: ligiarojas7@gmail.com

${ }^{3}$ Médico e Cirurgião. Universidade do Valle, Cali, Colômbia. Especialista em Cirurgia Vascular, Universidade da Costa Rica. Cirurgião independente. Ex-professor da Faculdade de Medicina da Universidade de Santiago de Cali. Colômbia. Correio eletrônico: medicosencali@hotmail.com

${ }^{4}$ Enfermeira Estudante de Mestrado em Enfermagem Ginecológica, Obstétrica e Perinatal da Universidade da Costa Rica. Trabalho independente. Costa Rica. Correio eletrônico: pilicardenas28@gmail.com

${ }^{5}$ Enfermeira Estudante de Mestrado em Enfermagem Ginecológica, Obstétrica e Perinatal da Universidade da Costa Rica. Trabalho independente. Costa Rica. Correio eletrônico: jennygo78@gmail.com
} 


\section{Revista Electrónica Enfermeria Actual en costa Rica}

\section{www.revenf.ucr.ac.cr}

\section{INTRODUCCIÓN}

Las venas varicosas se definen como venas superficiales elongadas, dilatadas anormalmente tortuosas ${ }^{1}$ : en el caso de mujeres durante el embarazo, es un problema de salud no reconocido por los expertos como una patología que provoca severas consecuencias, por su rápida regresión durante el puerperio ${ }^{2}$. Sin embargo, es de hacer notar que este problema produce en la gestante mucha disconformidad y dolor, por lo cual pueden ser tratadas cuando están en una fase aguda en cualquier momento del embarazo, lo cual disminuiría o desaparecería los síntomas, hasta prevenir la ocurrencia en embarazos posteriores o disminuir el problema.

En cuanto a la incidencia de las venas varicosas reticulares y telangiectasias, alrededor de la mitad de la población mundial las presenta en miembros inferiores, esto afecta al 50-55\% de las mujeres; en el caso de venas varicosas mayores y más visibles, afectan a menos de $1 / 4$ de la población, mientras que -en el caso de las mujeres- entre el 2 $-25 \%$ mencionan una prevalencia mundial de $2-56 \%$. Al respecto, "los investigadores han estado observando la correlación entre el embarazo y la enfermedad varicosa durante mucho tiempo y el aspecto de las dilataciones venosas de miembros inferiores o en los pechos de las mujeres en edad reproductiva se considera un signo de embarazo, y algunas mujeres atribuyen la aparición de varices con el embarazo y el empeoramiento de embarazos sucesivos" (traducción de los autores).

La incidencia de las venas varicosas aumenta con la edad con mayor prevalencia en mujeres (5:1), máxime en mujeres caucásicas, asociada a historia familiar, embarazo, obesidad, postura (de pie o sentado por largos periodos), ingesta de contraceptivos orales, uso de terapia hormonal de reemplazo ${ }^{1}$. Las mujeres multíparas tienen mayor riesgo de desarrollar venas varicosas en el tiempo, sin considerar la ganancia de peso asociada al embarazo; no obstante, las mujeres obesas son tres veces más propensas a desarrollarlas, aspecto que no ha sido observado en hombres ${ }^{4,5}$. Las úlceras asociadas a las venas varicosas afectan solamente al $1 \%$ de la población ${ }^{4,6}$ - en los Estados Unidos - donde el gasto excede en US\$1 billón anualmente.

En torno al tema, en un estudio sobre embarazo y venas varicosas en miembros inferiores, factores de riesgo y prevalencia ${ }^{3}$, en el que se consideró todo tipo de venas varicosas, de 352 mujeres embarazadas estudiadas, seleccionadas al azar durante 14 meses, la prevalencia de la enfermedad varicosa fue de $72,7 \%$ (256 mujeres embarazadas), lo cual contrasta con el 27,3\% (96) de las mujeres embarazadas que no presentaron esta condición de salud.

Respecto de la etiología, las venas varicosas primarias ocurren debido a la combinación del aumento en la elasticidad de las paredes y la incompetencia valvular ${ }^{7}$, cuyo aumento de la presión conduce a la dilatación progresiva. Los sitios más comunes de incompetencia son la safeno-femoral, entre la vena safena mayor y la vena femoral común; la unión de la safeno poplítea entre la vena safena menor y la vena poplítea detrás de la rodilla.

En cuanto a las sus causas, las venas varicosas secundarias ocurren debido al incremento de la presión en la vena superficial relacionada con patología de las venas profundas y/ o perforantes, mas también pueden ocurrir en asociación con malformaciones arterio-venosas (congénitas o adquiridas) ${ }^{1}$.

Aunque las venas varicosas están asociadas a una baja morbilidad, estas pueden afectar la calidad de vida y secundariamente la apariencia cosmética. Millones de personas buscan atención médica anualmente para mejorar la apariencia cosmética ${ }^{8}$ de esta condición. No obstante, independientemente de la apariencia, tiene mayor 
relevancia el tratamiento temprano y sintomático, caracterizado por dolor, pesantez de miembros inferiores, picazón, hinchazón, calambres, hormigueo, cambios en la coloración de la piel, edema ${ }^{1,4,9}$

Respecto de los factores de riesgo, la literatura ha demostrado en diversos estudios que existe una fuerte relación con el factor familiar y pocos casos de desorden congénito (se describe el síndrome Klippel-Trenaunay y Chuvash policitemia); sin embargo, no se han identificado los genes relacionados con la presencia de las venas varicosas ${ }^{4}$

El embarazo presenta un estado vascular muy particular y diferente, dado que muchos de los efectos son reversibles y acumulativos ${ }^{10}$. Un factor que contribuye a la presencia de venas varicosas en el embarazo es la obstrucción mecánica del flujo venoso en la pelvis, lo cual incrementa el volumen de sangre circulante, además de los efectos hormonales ${ }^{11}$ que causan dilatación del músculo liso con una inhibición de la contractilidad. Lo anterior se combina para dilatar los anillos valvulares venosos, en relación con la edad de la mujer, en las embarazadas más jóvenes generan mayor sensibilidad a los efectos de las venas varicosas y aumentan la tendencia a presentarse más temprano 5 .

Por otro lado, se asume que la progesterona tiene efecto en el desarrollo de la enfermedad varicosa en el embarazo $^{12}$. Al respecto, en un estudio realizado por Lenković, et al. ${ }^{13}$, en el año 2009 , cuyo objetivo fue investigar la contribución de la progesterona en el desarrollo de venas varicosas primarias sobre miembros inferiores durante el embarazo, se determinó los niveles de progesterona en 50 primíparas en la semana 14 de gestación, cuyos resultados fueron comparados con los obtenidos en un grupo control de 25 primíparas sin enfermedad varicosa visible. La concentración de progesterona en mujeres embarazadas con venas dilatadas $(159.9+15.8 \mathrm{nmol} / \mathrm{L})$ fue significativamente más alto comparado con el grupo control $(159.9 \pm 15.8 \mathrm{nmol} / \mathrm{L}$ vs 40.4+1.6 nmol/L; $\mathrm{P}<0.0001)$. Estos hallazgos apoyan el efecto del factor hormonal en el desarrollo de esta morbilidad en mujeres (traducción de los autores)

En un reporte de caso realizado por Kawata et al. ${ }^{14}$, en el año 2014, mencionan un hallazgo incidental en una sonografía transvaginal en un embarazo temprano (seis semanas de gestación) realizado a una mujer de 31 años, en quien se notó la presencia de varices útero-ováricas. La resonancia magnética reveló venas ováricas dilatadas con ausencia infrarenal de vena cava inferior, así como la ausencia de venas iliacas comunes y externas: a pesar de esa condición, la gestación transcurrió sin complicaciones, con parto vaginal y postparto exitoso. Los autores afirman que la ausencia de vena cava inferior y várices pélvicas puede ocurrir en mujeres con antecedentes de cirugía neonatal. Aunque el riesgo de ruptura de las várices útero-ováricas sigue siendo poco clara, el parto vaginal se logra de forma segura.

Otros estudios ${ }^{15}$ se han enfocado hacia la asociación de mujeres embarazadas con venas varicosas de extremidades inferiores y el posible riesgo de resultados adversos al nacer como: diferentes anormalidades congénitas en sus hijos. Sin embargo, los investigadores señalaron que no hay una asociación que marque un peligro obvio para los fetos.

Un aspecto relevante y objetivo de esta revisión son las opciones de tratamientos que se ofrecen a las mujeres gestantes: un elemento básico en el tratamiento crónico de la insuficiencia crónica venosa es la terapia compresiva, efectiva para el alivio de los síntomas; no obstante, también se ofrece a la población en general tratamientos quirúrgicos para el reflujo venoso. El propósito de la cirugía es eliminar el reflujo y corregir la 
patología hemodinámica venosa. Se ha comparado ambos tratamientos para eliminar el reflujo venoso en un ensayo clínico controlado: os resultados quirúrgicos mostraron mayor significancia de mejoría clínica en la severidad de la enfermedad y calidad de vida comparado con los pacientes sometidos a terapia compresiva solamente ${ }^{6}$.

Respecto de la intervención para el tratamiento de venas varicosas presentes en miembros inferiores en la mujer gestante, Akinloye, Justus ${ }^{16}$ llevaron a cabo cuatro ensayos de tres tratamientos diferentes: en uno, se dio cápsulas de rutósido en los últimos tres meses de embarazo, quienes mostraron una mejoría en los síntomas en comparación con el placebo (RR 0.54 95\%CI 0.32, 0,89), por ejemplo, disminuyó la circunferencia del edema del tobillo a las 36 semanas de gestación después de ocho semanas de tratamiento, mientras que las mujeres que recibieron placebo tuvieron un pequeño aumento de los síntomas. En otro ensayo, las mujeres con edema de tobillo tuvieron una pequeña reducción no significativa en el volumen inferior de la pierna a la que se aplicó compresión neumática externa intermitente por 30 minutos. En el otro ensayo, las medias de compresión redujeron profilácticamente la aparición de síntomas en las piernas, pero no las venas varicosas (RR 0,74; 95\% IC $0.59,0.93)$. La reflexología linfática se estudió en muy pocas mujeres. En las conclusiones mencionan que los rutósidos parecen aliviar los síntomas de la insuficiencia venosa al final del embarazo. Sin embargo, no se sabe si el fármaco es seguro durante la gestación. La compresión neumática externa parece reducir la hinchazón del tobillo y las medias de compresión, los síntomas de las varices.

Por otro lado, se menciona que las "opciones de tratamiento oscilan entre manejo conservador a través de los cambios de vida con el uso de terapia de compresión con medias compresivas a procedimientos más invasivos, incluyendo el tratamiento quirúrgico, la ablación térmica endovenosa, y la escleroterapia ${ }^{6}$.

En cuanto a la terapia de compresión, alivia los síntomas, pero los resultados a largo plazo -la recurrencia y la prevención de venas varicosas- no han sido apoyados por la evidencia. Por otra parte, "la evidencia varía respecto de la eficacia de procedimientos más invasivos en los resultados a largo plazo en cuanto a la recurrencia de venas varicosas y reflujo. Todos los pacientes deben ser educados acerca de los cambios en el estilo de vida y sobre sus opciones de tratamiento".

Respecto de la educación en salud, los profesionales en enfermería ginecoobstétrica, como miembros de un equipo de salud, son el personal idóneo para llevar a cabo esta tarea, razón por la que necesitan entender los diferentes tipos de opciones de tratamiento de pacientes con grados de variación de las venas varicosas. Además, deben realizar una evaluación inicial de las mujeres embarazadas sospechosas de sufrir esta alteración a la salud, sobre todo porque algunas son asintomáticas y solo pueden requerir el uso de un tratamiento conservador para evitar un mayor agravamiento y la progresión de la enfermedad. Para valorar las mujeres gestantes sintomáticas ${ }^{6}$, los profesionales deben actualizarse en relación con las pruebas iniciales disponibles y diversidad de tratamientos tanto conservadores como más invasivos con el fin de orientar a esas mujeres y remitirlas al profesional competente para su debida y oportuna atención.

El objetivo de esta revisión narrativa fue realizar una síntesis de los aspectos generales de las venas varicosas en miembros inferiores y su tratamiento durante el embarazo. 


\section{Revista Electrónica Enfermeria Actual en costa Rica}

\section{MATERIALES Y MÉTODOS}

Se elaboró una revisión de literatura internacional de investigaciones publicadas en las bases de datos electrónicas PubMed, Biblioteca Cochrane y EBSCO Host. No se aplicó ninguna restricción de lenguaje, mas sí un límite temporal de nueve años (2009-2017). La selección de los artículos se realizó en función de los siguientes criterios: estudios con metodología cuantitativa, revisiones sistemáticas cuantitativas, estudios observacionales, ensayos clínicos aleatorizados y no aleatorizados. Se excluyó estudios cualitativos y los no accesibles a texto completo.

Se utilizó las siguientes palabras clave del Medical Subject Headings (MeSH): "varicosities and pregnant", "treatment for varicose veins", prevalence and varicosities veins" "lower limb varicosities veins and pregnant" "pregnant women and varicose veins" "varicose veins medical treatment and pregnancy" "varicose disease and pregnant and prevention" "pregnancy and lower limb varicose veins" y sus equivalentes en español.

La búsqueda se dividió en las siguientes variables de interés: tratamiento médico conservador y tratamiento médico más invasivo incluyendo cirugía, factores de riesgo de la enfermedad varicosa durante el embarazo, sintomatología y diagnóstico.

De cada uno de los artículos seleccionados, se extrajo los siguientes datos: referencia, tipo del estudio, base de datos, tipo de intervención, tema de interés y resultados.

Del total de 50 referencias bibliográficas, se seleccionó seis documentos que contenían las variables de interés: una guía clínica, dos artículos de revisión, una revisión sistemática, un ensayo clínico, un estudio epidemiológico (de prevalencia).

\section{Consideraciones éticas}

Este estudio forma parte de un proyecto avalado por la Vicerrectoría de Investigación de la Universidad de Costa Rica con el código N. ${ }^{\circ}$ 421-B6-A25. Se respetó la autoría de los documentos citados.

\section{RESULTADOS}

\section{La enfermedad varicosa durante el embarazo}

La presencia de venas varicosas durante el embarazo es una enfermedad que afecta alrededor del $40 \%$ de las mujeres $^{9}$ : en torno al tema, estudios epidemiológicos señalan que las venas varicosas del sistema venoso superficial de las extremidades inferiores se desarrollan frecuentemente en mujeres y con mayor frecuencia durante el embarazo ${ }^{13}$. Por tal motivo, es importante que el abordaje en ese periodo sea asumido por un equipo de profesionales en salud, brindado en servicios de medicina vascular ${ }^{17}$.

Respecto de la patogénesis todavía no ha sido bien clarificada ${ }^{13}$; algunos investigadores señalan que existe una incompetencia valvular, trombosis o elevada presión venosa que causa cambios en la circulación venosa y que se manifiesta en la piel $^{13}$, sin olvidar que el sistema venoso altera su función en el embarazo y que los cambios son funcionales y estructurales ${ }^{12}$. 
En otras teorías se menciona que el componente hormonal, específicamente la progesterona y los estrógenos provocan numerosos efectos sobre el sistema vascular mediante los receptores de estas hormonas ubicados en las venas safenas y aunque no está clara la función que tienen, algunos autores afirman que pueden provocar dilatación venosa e insuficiencia valvular en el embarazo, al provocar relajación del músculo liso y de las fibras de colágeno ${ }^{12,18}$. Las venas varicosas se encuentran más comúnmente en mujeres embarazadas con altas concentraciones de esta hormona; aunque el nivel varía individualmente entre las mujeres en general (embarazadas y no embarazadas), el porcentaje normalmente se encuentra entre $1 \mathrm{nmol} / \mathrm{L}$ y $20 \mathrm{nmol} / \mathrm{L}$, rango que dependerá del ciclo menstrual, más alto durante la fase lutea y en el embarazo. De igual forma, los estrógenos tienen un efecto similar y, además, pueden causar un deterioro en la conexión y síntesis de las fibras de colágeno y afectar la síntesis de prostaglandinas y óxido nítrico, una molécula con un fuerte efecto vasodilatador ${ }^{12}$. Según lo anterior, la progesterona relaja las paredes musculares de los vasos sanguíneos lo que dificulta mover la sangre desde la parte inferior del cuerpo hacia el corazón. El factor mecánico, o sea, la compresión de las venas iliacas y la cava inferior debido al peso del útero en crecimiento ejerce presión y favorece la presencia de varices en miembros inferiores ${ }^{9,13}$.

A medida que el bebé crece y el útero se agranda, las venas se vuelven aún más prominentes, proceso que -a su vez- puede conducir a insuficiencia venosa y edema de la pierna. La estasis venosa y su consecuente hipertensión en el segmento insuficiente provocan que la sangre se acumule en las piernas, lo cual genera que el líquido de las venas ingrese a los tejidos de los pies y tobillos con la concomitante hinchazón?

\section{Factores de riesgo de la insuficiencia venosa en miembros inferiores durante el embarazo}

Entre los factores de riesgo que acompañan la enfermedad varicosa se menciona un aumento en el volumen de sangre circulante, expansión del útero, aumento de peso, cambios hormonales y reducción en la actividad física, así como cambios plasmáticos en el sistema venoso y un aumento en el nivel de fibrinógeno y los factores de coagulación VII, VIII, IX y X y el factor de Von Willenbrand.

La cesárea es también un factor de riesgo para la trombosis venosa. Los factores familiares están asociados con la herencia ${ }^{19}$ en la formación de cambios varicosos e insuficiencia venosa en ambos sentidos, dominante y recesivo, y también relacionada con el sexo. De Barros ${ }^{3}$ menciona que Dindelli et al. encontraron el riesgo relativo 6.2 veces más alto si hay presencia de enfermedad venosa en mujeres embarazadas con historia familiar positiva; por otro lado, el mismo autor menciona que Cornu-Thenard et al. reportaron que el riesgo de desarrollar venas varicosas fue de $90 \%$ cuando ambos padres lo presentaban y el $62 \%$ cuando lo presenta solo la madre.

Entre otros factores que afectan el desarrollo de la insuficiencia venosa durante el embarazo, se pueden distinguir: tipo de trabajo (de pie, sentado, en posición forzada y vibración) ${ }^{12,19}$, intervalo entre embarazos (determinando la posibilidad de regeneración fisiológica del sistema). En el caso de las mujeres que han estado embarazadas más de una vez, el riesgo de desarrollar venas varicosas y otras insuficiencias venosas se duplica ${ }^{12}$, por lo tanto, la multiparidad es un factor de riesgo muy importante, se ha observado 3.8 veces mayor riesgo en la segunda gesta, 1.2 veces más en primípara comparada con nulípara $3,9,12,13,19$. La edad también influye en la prevalencia de la enfermedad varicosa, De Barros ${ }^{3}$ señala que existe predominio en el grupo poblacional comprendido entre 20 y 29 años. 


\section{Signos y síntomas}

En el $70-80 \%$ de las mujeres que desarrollan problemas con venas varicosas en el embarazo, los síntomas aparecen durante el primer trimestre. El $80 \%$ puede verse afectado por edema en las piernas que se resuelve después del nacimiento; el dolor es el síntoma más común ${ }^{9,17,19}$; pero también los estudios reportan calambres nocturnos, pesadez, prurito, cambios de coloración en la piel alrededor de la vena varicosa ${ }^{9,17}$, la cual mejora a los tres o cuatro meses después del nacimiento ${ }^{9}$. Otros autores también mencionan la presencia de eczema causada por insuficiencia venosa crónica, trombosis venosa superficial y úlceras venosas ${ }^{17}$.

\section{Diagnóstico}

Es importante realizar la valoración clínica con la mujer de pie y efectuar una historia clínica. Se puede instrumentar mediante doppler, ultrasonido dúplex preoperatorio, venografía, resonancia magnética venográfica. Para casos severos donde la incompetencia venosa no es en miembros inferiores se recomienda una venografía tomográfica $^{19}$.

Se han diseñado una serie de cuestionarios que se aplican a la mujer para tener una panorámica más certera y llevar a cabo el diagnóstico. A continuación, se describe estos cuestionarios ${ }^{19}$ :

1. Cuestionario (VCSS):Aberdeen Varicose Vein

2. Cuestionario de calidad de vida (QoL)

3. Cuestionario (CIVIQ-20): para determinar la insuficiencia venosa crónica

4. El cuestionario (VEINES): de epidemiología y estudio económico de insuficiencia venosa

\section{Manejo del tratamiento de venas varicosas durante el embarazo}

\section{Tratamiento conservador}

El manejo del tratamiento de primera línea para venas varicosas durante el embarazo es conservador: se recomienda el uso de medias compresivas, por ejemplo, en el Reino Unido, se clasifica en tres grados (I, II y III) para indicar los mmHg de compresión según sea el caso que se presente ${ }^{9,19,20,21}$. Las medias compresivas tienen el rol de aliviar la hinchazón en miembros inferiores asociado a venas varicosas y el prurito ${ }^{9,17}$. También es importante dar información a las mujeres embarazadas sobre el efecto del embarazo en las venas varicosas ${ }^{21}$, para que el profesional de salud (médicos, médicos especialistas y enfermera (os) ginecoobstetras y perinatales) se mantenga actualizado sobre el tema para que pueda guiar a las mujeres durante todo su proceso gestacional y posterior aparición de este problema de salud.

Otras recomendaciones importantes consisten en elevar las extremidades por la noche y durante los periodos de descanso durante el día, de modo que se evite la dependencia estática de las piernas y el control del peso corporal. En caso de estasis venosa y síntomas graves de varicosis que no se pueden controlar con medidas conservadoras, está indicada una intervención quirúrgica limitada ${ }^{20}$. 
Otra medida importante de resaltar es que las mujeres descansen sobre su lado izquierdo ya que la vena cava inferior está a la derecha, por lo que al descansar sobre su lado izquierdo alivia la compresión del peso del útero y disminuye la presión sobre las venas de las piernas y los pies, además de que realicen ejercicios de flexión suave del pie para estirar los músculos de la pantorrilla9, así como practicar la reflexología. El concepto sugiere que todos los órganos y glándulas del cuerpo tienen puntos reflejos correspondientes en los pies, las manos y los oídos; por lo que al estimular estos puntos potencialmente se proporciona alivio del dolor y de los síntomas e induce la relajación sin efectos secundarios dañinos. La reflexología se puede utilizar para afecciones específicas en miembros inferiores y el edema generalizado, ya que moviliza los fluidos de los compartimentos extravasculares sin afectar los intravasculares ${ }^{22}$.

Risch (1978) mencionado por Smyth $^{9}$ señala que la inmersión en el agua es otro tratamiento que se ha utilizado ampliamente: para el tema de interés de esta revisión, importa la posibilidad de reducir el edema de la pierna, dado que esta práctica puede ayudar a mejorar el retorno venoso y linfático, primero a los muslos, luego a los vasos de la cavidad abdominal y finalmente a los grandes vasos de la cavidad torácica y corazón, esto se debe a que la persona está sometida a presión de agua externa en una gradiente que en una profundidad relativamente pequeña excede la presión venosa.

Finalmente, Hayes (1999), mencionado por $\mathrm{Smyth}^{9}$, relata que el masaje de pies es otro ejemplo de intervención no farmacológica que ayuda a reducir el edema, funciona moviendo el líquido extravascular -sin alterar el intravascular- mediante un masaje en pies y piernas.

\section{Tratamiento invasivo}

En la literatura se encuentra una diversidad de tratamientos dirigidos a mejorar la enfermedad varicosa en general, tales como escleroterapia, la ablación con radiofrecuencia (RFA), ablación endovenosa láser (EVLA), terapia con vapor (Steam varicose system), la crioablación, ablación endovenosa química-mecánica; sistema de cierre venoso (Sapheon), cirugía abierta (ligadura de unión safenofemoral (SFJL), ligadura de unión safenopoplitea (SPJL), manejo hemodinámico conservador ambulatorio de las venas varicosas (CHIVA), ablación selectiva de varices ambulatoria bajo anestesia local (ASVAL) entre otros ${ }^{19}$.

Específicamente para la mujer embarazada no se recomienda ningún tratamiento intervencionista ${ }^{17,21}$ solo en casos excepcionales $^{21}$ : los tratamientos invasivos solo después de tres o cuatro meses después del nacimiento ${ }^{9}$.

A continuación, se presenta la tabla 1 que corresponde a un resumen de las referencias bibliográficas que responden a factores de interés en esta revisión antes descrito. Onida, Tristan y Davies ${ }^{19}$ elaboraron una revisión sobre el manejo de las modalidades de las venas varicosas; O'Flynn, Vaughan y Kelly ${ }^{17}$ presentan un resumen de la guía de práctica clínica sobre el manejo y diagnóstico de las varices venosas en los miembros inferiores; Smyth, Aflaifel, Bamigboye ${ }^{9}$ llevan a cabo una revisión sistemática cuantitativa sobre las intervenciones para la venas varicosas y el edema en la pierna durante el embarazo; por su parte, Lenkovic et al. ${ }^{13}$ desarrollaron un ensayo clínico cuyo objetivo fue investigar la contribución de la progesterona al desarrollo de venas varicosas primarias en miembros inferiores durante el embarazo; De Barros, Janeiro, de Amorin ${ }^{3}$ realizaron un estudio de prevalencia para verificar la enfermedad varicosa durante la gestación y, finalmente, se encontró la guía clínica de NICE que 
establece recomendaciones a los clínicos para el diagnóstico y manejo de las venas varicosas, la cual cuenta con un apartado específico para el embarazo. Véase tabla 1.

Tabla 1. Referencias bibliográficas que responden a factores de interés en la revisión

\begin{tabular}{|c|c|c|c|c|c|c|}
\hline $\begin{array}{l}\text { Factor de } \\
\text { interés / } \\
\text { Referencia }\end{array}$ & $\begin{array}{l}\text { Onida, Tristan, } \\
\text { Davies }(2013)^{19}\end{array}$ & $\begin{array}{l}\text { O'Flynn, Vaughan, } \\
\text { Kelly }(2014)^{17}\end{array}$ & $\begin{array}{l}\text { Smyth, Aflaifel, Bamigboye } \\
(2015)^{9}\end{array}$ & $\begin{array}{l}\text { Lenkovic et al. } \\
(2009)^{13}\end{array}$ & $\begin{array}{l}\text { De Barros, Janeiro, } \\
\text { de Amorin, } \\
\text { Miranda }(2010)^{3}\end{array}$ & NICE $(2013)^{21}$ \\
\hline $\begin{array}{l}\text { Influencia del } \\
\text { embarazo en las } \\
\text { venas }\end{array}$ & & $\begin{array}{l}\text {-Equipo de } \\
\text { profesionales para la } \\
\text { atención }\end{array}$ & $\begin{array}{l}\text {-Problema común. } \\
\text {-Embarazo se presume como el } \\
\text { mayor factor contribuyente } \\
\text {-Afecta alrededor del } 40 \% \text { de } \\
\text { gestantes. }\end{array}$ & $\begin{array}{l}\text {-Patogénesis no ha sido } \\
\text { bien clarificada. } \\
\text {-Progesterona y } \\
\text { estrógenos tienen } \\
\text { números efectos sobre el } \\
\text { sistema vascular. } \\
\text {-Es frecuente en el } \\
\text { embarazo. } \\
\text {-Prevalencia más baja en } \\
\text { mujeres de países } \\
\text { desarrollados }\end{array}$ & & \\
\hline $\begin{array}{l}\text { Factores de } \\
\text { riesgo }\end{array}$ & $\begin{array}{l}\text {-Obesidad } \\
\text {-Embarazo } \\
\text {-Historia familiar } \\
\text {-Postura }\end{array}$ & & -Multiparidad & -Multiparidad & $\begin{array}{l}\text {-Edad (mujeres } \\
\text { mayores a } 70 \text { años y } \\
\text { en el grupo entre 20- } \\
29 \text { años) } \\
\text {-Multiparidad } \\
\text {-Historia familiar } \\
\end{array}$ & \\
\hline $\begin{array}{l}\text { Signos y } \\
\text { síntomas }\end{array}$ & $\begin{array}{l}\text {-Dolor, pesadez, } \\
\text { hinchazón, prurito, } \\
\text { edema y cambios en la } \\
\text { piel }\end{array}$ & $\begin{array}{l}\text {-Cambios en la piel, } \\
\text { pigmentación, eczema, } \\
\text { dolor, úlceras venosas }\end{array}$ & $\begin{array}{l}\text {-Aparecen en el primer trimestre. } \\
\text { Edema, dolor, calambres nocturnos, } \\
\text { pesadez, prurito, cambios en la piel, } \\
\text { postura }\end{array}$ & & & \\
\hline Diagnóstico & $\begin{array}{l}\text {-Evaluación de la } \\
\text { paciente de pie } \\
\text {-Uso de doppler } \\
\text {-Aplicación de } \\
\text { cuestionarios } \\
\text {-Ultrasonido dúplex } \\
\text {-Venografía, resonancia } \\
\text { magnética, venografía } \\
\text { tomográfica }\end{array}$ & $\begin{array}{l}\text {-Evaluación con } \\
\text { ultrasonido doppler } \\
\text { dúplex }\end{array}$ & & & & \\
\hline $\begin{array}{l}\text { Tratamiento } \\
\text { conservador }\end{array}$ & $\begin{array}{l}\text {-Drogas no esteroides } \\
\text { antiinflamatorias } \\
\text {-Media compresiva } \\
\text {-Educación }\end{array}$ & -Medias compresivas & $\begin{array}{l}\text {-Educación, medias compresivas, } \\
\text { descanso, flexión suave del pie, } \\
\text { reflexología, inmersión en agua, } \\
\text { masaje de pies }\end{array}$ & & & $\begin{array}{l}\text { - Información, } \\
\text { medias } \\
\text { compresivas }\end{array}$ \\
\hline $\begin{array}{l}\text { Tratamiento } \\
\text { invasivo }\end{array}$ & & $\begin{array}{l}\text {-En embarazo, solo en } \\
\text { casos excepcionales }\end{array}$ & $\begin{array}{l}\text {-Se recomiendan } 3 \text { o } 4 \text { meses } \\
\text { después del nacimiento }\end{array}$ & & & $\begin{array}{l}\text {-No tratamiento } \\
\text { intervencionista, } \\
\text { solo en casos } \\
\text { excepcionales }\end{array}$ \\
\hline
\end{tabular}

Fuente: elaboración propia

\section{DISCUSIÓN}

Se demuestra que la enfermedad varicosa es común ${ }^{23}$ :en general, es una condición de salud que afecta a gran parte de la población mundial, tanto a hombres como mujeres, aunque mayormente en mujeres, cuyo caso más común se da en miembros inferiores. Desde los años 50, los investigadores se han interesado por el tema, por lo que han 
emitido recomendaciones de tratamiento para los diferentes grados de la enfermedad y no han dejado de lejos el interés en el tema correlacionado con el embarazo por los cambios importantes que se suscitan en el cuerpo y que de una $\mathrm{u}$ otra manera pueden favorecer la presencia de la enfermedad varicosa o intensificar el padecimiento si existiera antes del embarazo. No obstante, en la actualidad no se encuentran tratamientos novedosos, especialmente para las mujeres gestantes, que ayuden a lidiar con la presencia de venas varicosas y a prevenir complicaciones en embarazos posteriores.

En la literatura se ha identificado diferentes teorías que pretenden explicar la presencia de las venas varicosas durante el periodo gestacional, pero no hay una claridad contundente que satisfaga la explicación de esta morbilidad $^{13}$.

Por su parte, los factores de riesgo y la prevalencia suman importancia en el embarazo debido a que la edad y la multiparidad desempeñan un papel preponderante ${ }^{3,9,13,19}$. En las poblaciones jóvenes (20-29 años) alcanza la mayor presencia de venas varicosas en miembros inferiores que, de no tratarse a tiempo, se pueden suscitar verdaderas complicaciones posteriores. Respecto de la herencia familiar es un factor que también ha sido discutido por varios autores, sin embargo, hay controversia respecto de si influye o no en la morbilidad; no obstante, pareciera que sí tiene un peso importante en este padecimiento que, sumado a otros factores de riesgo como el estilo de vida, la obesidad, la postura ${ }^{19}$, alcanza mayores probabilidades de sufrirla.

Las recomendaciones de tratamiento que han evidenciado los estudios no guardan mucha diferencia a décadas pasadas, más bien las investigaciones han apuntado a mejorar las técnicas y crear nueva aparatología para llevar a cabo un mejor diagnóstico y tratamientos que sean mínimamente invasivos. Por su parte, para las mujeres embarazadas, desde los años 50 se han recomendado las medias compresivas solamente y a la fecha esto no se ha modificado ${ }^{9,17,19}$. Los autores de esta revisión consideran que es importante realizar nuevos estudios que den una mayor posibilidad de tratamiento a las mujeres embarazadas que permita cursar la gestación con comodidad y por el bienestar del futuro bebé. También es importante elaborar revisiones sistemáticas que ayuden a los profesionales de la salud a mantenerse actualizados en el tema para que de esta forma puedan proporcionar mayor educación en salud a las mujeres gestantes.

Actualmente, se está recomendando las terapias complementarias o alternativas para el tratamiento de las venas varicosas, haciendo la salvedad de que deben llevarse a cabo estudios rigurosos y robustos que demuestren la efectividad y seguridad tanto para la madre como para el feto. Dentro de estas terapias, se recomienda la reflexología, la inmersión en agua y el masaje9.

Respecto de los signos y síntomas, suelen aparecer desde el primer trimestre ${ }^{9,17,19}$. Los profesionales en salud deben abocarse a llevar a cabo una valoración profunda que determine desde el inicio el tratamiento a seguir, así como la educación que se le debe dar a la mujer, con la finalidad de prevenir complicaciones posteriores al nacimiento y acrecentar el padecimiento con embarazos futuros.

A partir de lo anterior, se demuestra que el abordaje a la mujer embarazada debe estar suscitado por un grupo de profesionales debidamente capacitados y coordinados para asegurar el éxito en el proceso por el que pasa la madre y el feto, sin dejar de lado la necesidad de seguir investigando sobre formas novedosas, efectivas y seguras para el tratamiento de la enfermedad varicosa durante el embarazo. 


\section{Revista Electrónica Enfermeria Actual en costa Rica}

\section{www.revenf.ucr.ac.cr}

\section{CONCLUSIÓN}

La enfermedad varicosa es un problema de salud que afecta a gran parte de la población mundial, especialmente a las mujeres, máxime en etapa de embarazo, en la cual debe prevalecer el abordaje dirigido hacia la prevención, proporcionada por un equipo de profesionales en salud para disminuir la presencia de esta morbilidad o, en su defecto, minimizar la sintomatología. Existe una gran necesidad de llevar a cabo estudios rigurosos y robustos que demuestren cuáles son las mejores medidas profilácticas, más novedosas y de tratamiento desde el inicio del embarazo, además de prevenir la aparición posterior, de manera que esté asegurada la salud de la mujer y, consecuentemente, la de su bebé.

\section{CONFLICTO DE INTERESES}

Los autores declaran que no tienen conflicto de intereses personales, sociales, económicos, laborales ni de ninguna otra índole.

\section{REFERENCIAS BIBLIOGRÁFICAS}

1. Winterborn R y Smith F. (2010) Varicose veins. Surgery 28 (6) 259-262.

2. Siegler J. (1939) The treatment of varicose veins in pregnancy. American Journal of Surgery New Series Vol. XLIV, (2) 403-408.

3. De Barros N, Janeiro MC, De Amorim J, Miranda F. (2010) Pregnancy and lower limb varicose veins: prevalence and risk factors. J.Vasc Bras. 2010, 9 (2) 29-35.

4. Vandy F, Wakfield T. (2012) Varicose veins: evaluating modern treatments, with emphasis on powered phlebectomy for branch varicosities. Interv. Cardiol. 4(5), 527-536.

5. Dijkstra M, Khin N, Coroneos J, Hazelton S, Lane R. (2014) The effect of pregnancy on venous valve repair to the sapheno-femoral junction for varicose veins. Obstetric Medicine 7 (2), 84-89.

6. Zhang S. \& Melander S. (2014) Varicose Veins: Diagnosis, Management and Treatment. The Journal for Nurse Practiotioners-JNP, 10, Issue 6, 417-424.

7. Boivin P, Cornu-Thenard A, Charpak Y. Pregnancy induced changes in lower extremity superficial veins: an ultrasound scan study. Journal of Vascular Surgery. 2000; 32(3): 570-574.

8. Gloviczki P, Comerota A, Dalsing M, Eklof BoG, Gillespie D, Gloviczki M. et al. The care of patients with varicose veins and associated chronic venous diseases: Clinical practice guidelines of the Society for Vascular Surgery and the American Venous Forum. Journal of Vascular Surgery. 2011; 53(5) Supplement: 2S-48S. 
9. Smyth R, Aflaifel N, Bamigboye A. (2015) Interventions for varicose veins and lego oedema in pregnancy. The Cochrane Library, Issue 10

10. Sparey C, Haddad N, Sissons G, Rosser S, Cossart L. The effect of pregnancy on the lower limb venous system of women with varicose veins. Eur J Vasc Endovasc Surg. 1999; 18: 294-299.

11. Cordts P, Gawley T. Anatomic and physiologic changes in lower extremity venous hemodynamics associated with pregnancy. Journal of Vascular Surgery. 1996; 24 (5): 763-767.

12. Ropacka-Lesiak M, Kasperczak J, Breborowicz CH. Risk factors for the development of venous insufficiency of the lower limbs during pregnancy. Ginekol Pol. 2012; 83(12): 939-42.

13. Lenković M, Čabrijan L, Gruber F, Batinac T, Manestar-BlazićT, Stanić Z, Stašić A. (2009) Effect of progesterone and pregnancy on the development of varicose veins. Acta Dermatovenerol Croat, 17(4), 263-267.

14. Kawata E, Kondoh E, Kawasaki K, Baba T, Ueda A, Kido A \& Konishi I. (2015) Utero-ovarian varices and absent inferior vena cava in pregnancy. J. Obstet. Gynaecol 41 (4), 631-634. Doi: 10.1111/jog.12599.

15. Ferenc Bánhidy, Nándor Ács, Erzsébet H. Puhó, Andrew E. Czeizel. Varicose veins of lower extremities in pregnant women and birth outcomes. Cent.Eur J Public Health. 2010; 18(3):161-168.

16. Akinloye A, Justus G. (2006) Interventions for leg edema and varicosities in pregnancy. What evidence? European Journal of Obstetrics \& Gynecology and Reproductive Biology 129, 3-8.

17. O’Flynn N, Vaughan M, Kelly K. Diagnosis and management of varicose veins in the leg. NICE guideline. British Journal of General Practice. 2014.

https://www.ncbi.nlm.nih.gov/pmc/articles/PMC4032011/

18. Mashiah A, Berman V, Thole HH, Rose SS, Pasik S, Schwarz H, et al. Estrogen and progesterone receptors in normal and varicose saphenous veins. Surgery. 1999; 7(3): 327-331.

19. Onida S, Tristan RA, Davies A. Varicose veins and their management. Surgery. 2013; 31:211-217.

20. Greenstone SM, Craig E, Massell T. Management of varicose veins during pregnancy. Calif Med. 1957; 87(6): 365-367.

21. NICE. Varicose veins: diagnosis and management. NICE. Org.uk. 2013.

22. Mollart L. Single-blind trial addressing the differential effects of two reflexology techniques versus rest, on ankle and foot oedema in late pregnancy. Complement Ther Nurs Midwifery. 2003; 9(4): 203-208. 
23. Sell H, Vikatmaa P, Albäck A, Lepäntalo M, Malmivaara A, Mahmoud O, Venermo M. (2014)

Compression Therapy Versus Surgery in the Treatment of Patient with Varicose Veins: A RCT. European Jounal of Vascular and Endovascular Surgery 47 Issue 6, 670-677. 